\title{
Evaluation and Use of an Index of Oral Health Status
}

\author{
W. Paul Lang, DDS, MPH; Wenche S. Borgnakke, DDS, PhD; George W. Taylor, DMD, DrPH; \\ Marilyn W. Woolfolk, DDS, MPH; David L. Ronis, PhD; Linda V. Nyquist, PhD
}

\begin{abstract}
Objectives: The goals of this investigation were (1) to evaluate the Oral Health Status Index in relation to demographic characteristics, socioeconomic status, and preventive behaviors of an adult population; and (2) to understand how individual index components performed as indicators of oral health status compared to the composite index. Methods: The Oral Health Status Index (OHSI) was used on a probability sample of adults, aged 18-93 years, living in the Detroit tricounty area. Data were collected on 509 subjects via in-home dental examinations. Bivariate and multivariate analyses were used to compare the OHSI and its components, including decayed, missing, and replaced teeth, free ends, and moderate and severe periodontal disease measures. Results: The mean OHSI score for subjects was 77.3 ( $\mathrm{SE}=1.83$ ) with a range of -8.0 to 100.0. In regression analyses, OHSI scores were positively correlated with subjects' education level, self-rated oral health scores, and frequency of dental checkups and negatively correlated with age, nonwhite race, and smoking. Of the index components, missing teeth performed well as an indicator of oral health status. Missing teeth were positively correlated with age, nonwhite race, and smoking and negatively correlated with education level, self-rated oral health, and use of Medicaid. About 53 percent of variance in OHSI scores was explained by the multivariate models, compared to 46 percent for missing teeth. Conclusions: Choosing an indicator of oral health status likely will depend upon the characteristics of the population to be studied. As a composite measure of oral health status, the OHSI performed acceptably; however, missing teeth, an index component, also worked well. Continued evaluation of the OHSI is warranted. [J Public Health Dent 1997;57(4):233-42]
\end{abstract}

Key Words: oral health status, index, construct validation, population studies.

The measurement of oral health is recognized as a critical feature of numerous dental activities: describing normal biologic processes, understanding the natural history of disease, testing hypotheses regarding preventive agents, and planning and evaluation of health services $(1,2)$. Historically, most instruments developed for this activity were derived from a disease-based theoretical model and applied by providers using quantitative methods of measurement (3). Currently, conceptual models of oral health and instruments are focusing on a biopsychosocial perspective, incorporation of patients' points of view, and qualitative measurements $(3,4)$. In the future, instruments or indices of oral health status likely will combine elements of both models with clinically observed elements (provider perspectives) and self-reported measures (patient perspectives). To date, no single index has all these elements. Nonetheless, understanding which elements to include or how various elements interact is useful.

Development and evaluation of composite clinical instruments have been somewhat dormant, at least as measured by published reports, since the early 1980s. The Oral Health Status Index (5) was a measure developed at that time, but it subsequently was never evaluated extensively. While nearly two decades have passed since its development, this index still deserves scrutiny, as it has some appealing qualities: a limited set of weighted variables that derive a single measure of health and a straightforward method of data collection. The theoretical range of OHSI scores is from -53.9 (every tooth has severe bone loss and frank decay) to 0 (edentulous without tooth replacement) to 100 (no frank decay, missing teeth, or bone loss). However, questions quickly arise about its current usefulness given changes in disease trends, its potentially limited set of clinical indicators, and the absence of evaluation. Thus, the purpose of this report is to evaluate the Oral Health Status Index. While few guides for evaluation are available, the ideal index should possess certain characteristics such as simplicity, objectivity, validity, reliability, and sensitivity (1). Of these characteristics, validity is particularly important

Three types of validity - criterion, content, and construct validity - have been described by Kaplan and coworkers (6). For oral health status, there is no superior criterion for comparison with the OHSI, a fact that limits evaluation. Content validity can be assessed by determining if the OHSI adequately represents the domain of clinically observed oral health. Content validity is affected by the theoretical construct chosen for index development (7) and the components used within the index (3). Disease trends also can affect content validity as the importance of a particular component like caries may change with time. Some questions are: what constitutes clinically observed oral health status, how well does the OHSI represent this status, and would one or more of the OHSI components represent oral

Send correspondence and reprint requests to Dr. Lang, Department of Periodontics/Prevention/Geriatrics, University of Michigan, School of Dentistry, 1011 North University, Ann Arbor, MI 48109-1078. E-mail: wplang@umich.edu. Dr. Ronis is affiliated with the University of Michigan Institute for Social Research and School of Nursing and US Department of Veterans Affairs. Drs. Borgnakke, Taylor, Woolfolk, and Nyquist are at the University of Michigan School of Dentistry in Ann Arbor. Supported by NIDR grant \#DE10145. Poster presented at the annual meeting of the American Association of Public Health Dentistry at Las Vegas, NV, October 1995. Manuscript received: 4/12/96; returned to authors for revision: 5/29/96; accepted for publication: 6/19/97. 
health status as well as or better than the OHSI.

Determining what constitutes clinically observable oral health status may be considered difficult by some and impossible by others, particularly in the context of a composite index. However, four approaches that span about 30 years are considered. Bulman (8) proposed a system called Oral Health Grading that was an early attempt to describe the total effect of all disease processes acting in the mouth. He recognized the need for a simple system, proposing three major areas for evaluation: dental, periodontal, and prosthetic. Orthodontics was considered, but excluded to maintain index simplicity. The dental component considered carious and missing teeth. The periodontal component contained observations about periodontal pocketing and calculus deposits. For example, an individual with pocketing in excess of $3 \mathrm{~mm}$ around more than two teeth and gross general calculus coupled with gross chronic inflammation was graded as having poor periodontal health.

Prosthetic grading either was acceptable or unacceptable - the former described as either no dentures or bridges required or dentures and/or bridges wom and functioning satisfactorily. With this grading system, 17 categories were possible; these were eventually reduced to seven, with one being good oral health and seven being poor oral health. Grading within each component - good, fair, poor was felt to be arbitrary by some (9), and the index did not appear to lend itself well to statistical analysis. However, this grading system can be viewed as the beginning of operationally defining index content to include decayed and missing teeth, periodontal health, and presence and satisfactory functioning of prostheses.

Nikias and colleagues (10) developed an oral health index based on the ranking of oral status profiles by dental professionals. Index development was guided by empirical distributions of observed oral conditions in a large population and the desire to be maximally comprehensive (9). Profiles of oral status initially included edentulousness, number of missing and unreplaced teeth, levels of gingival and periodontal conditions, percent of decayed teeth, oral hygiene status, number of sound and untreated teeth, number of filled teeth, and number of replaced teeth. Grading occurred in each category. To create a useful and manageable number of profiles, dimensions were eliminated and grading was collapsed. Four dimensions were retained: missing teeth and unreplaced teeth, gingival and periodontal disease, decayed teeth, and oral hygiene status. Grades were collapsed to make the profiles more manageable. Ultimately, 42 profiles were ranked by dental professionals to create a range of profiles. The most healthy profile was zero to two missing and unreplaced teeth, no periodontal disease, no decayed teeth, and good oral hygiene. The least healthy profile was nine or more missing and unreplaced teeth, high periodontal disease, some decayed teeth, and poor oral hygiene. This method created a montage of characteristics in which it was difficult to identify areas of change or to discern how scoring high on one attribute might affect scoring low on another attribute (7). Mathematical computation of a population score also appeared to be difficult. However, the essential elements of oral health status identified by these investigators were missing and unreplaced teeth, presence of periodontal disease, decayed teeth, and oral hygiene level.

The index under investigation in this report - the Oral Health Status Index - was devised by Marcus, Koch, and Gershen (5,7,11-13) for planning and monitoring the outcome of dental care services for adult populations. A pair-preference approach was used to derive the index in which clinicians were asked to determine what constituted good oral health on a large set of cases comparing two at a time. Beginning with 24 variables representing diverse factors impinging on oral health, the set of factors was decreased to 18 , then eight, and ultimately to six: severe and moderate bone loss, missing teeth, free ends (loss of at least all three molars in a quadrant), decayed/fractured teeth, and replaced teeth. The ultimate selection of these six indicators appeared to be based on several considerations. Developers wanted to be able to represent the interaction and effects of the two major oral diseases - caries and periodontal disease. Severe and moderate periodontal disease, missing teeth, and decay were variables ranked highest by the clinicians. Intui- tion suggested no more than five or six easily measured variables, and repeated analyses indicated reasonably good explanatory power with the selected variables (7).

Some concerns with the OHSI can be readily identified. The index was developed nearly 20 years ago when disease patterns and practitioners' perceptions of these patterns were quite different than those of today. The study population from which the simulated cases were derived displayed disease conditions in excess of current levels. For example, 88 percent of subjects had some decay, 95 percent had one or more missing teeth, and 50 percent had moderate or severe bone loss (12). Braun (14) also observed that the index was developed using healthy adults, and might not be sensitive to extraoral and age-related dental variables. On the other hand, the conditions included in the OHSI still occur today, likely are modestly prevalent in some disadvantaged or older age groups, and would surely be included in a clinical index if derived now. One could argue that variables such as temporomandibular disorders, occlusal function, or endodontic treatment should be included as they have assumed more importance in a contemporary view of clinical oral health. The prevalence and significance of such variables will need to be considered. The OHSI, despite its age and limited number of components, still accounts for caries, periodontal disease, missing teeth, and replaced teeth.

Multiattribute utility theory and an expert panel modeling methodology were used to develop a recent measure of oral health status described in an unpublished paper (Sainfort F, Zimmerman DR, Booske BC, Wickeham D. Oral health outcomes measurement using multiattribute utility theory. Unpublished manuscript, 1994). An expert panel defined oral health in terms of eight issues: periodontal status, restorative status, pain, oral pathology, temporomandibular disorder, cosmetics, occlusion, and habits. Thirty-nine components were identified for the eight issues. For example, the issue of periodontal status contained six components: quadrant plaque index, bleeding point index, periodontal pocket index, mobility, bone loss, and loss of attached gingiva. Restorative status contained $10 \mathrm{com}$ - 
ponents, including missing teeth, caries, fractured teeth needing restoration, recurring caries around restorations, and open contacts. Multiattribute utility theory was used to assign issue and component weights, with the result being a potential score that could range from 0 to 100,100 being perfect health. The index was computed on about 4,000 individuals to investigate its potential usefulness. Of the 39 components, 15 were observed in 10 percent or less of the population. The more frequently observed conditions included plaque, bleeding, periodontal pockets, bone loss, missing teeth, decayed teeth, cosmetics (malalignment, spaces), and occlusion (function, esthetics). This index appears to address many contemporary oral health issues, but may contain too many components. Measuring all 39 components would likely be time consuming.

Some elements are common among the four preceding attempts to quantify clinically observed oral health status. Counts of decayed and missing teeth were used by all measures, as was measurement of periodontal pocketing or bone loss. Prosthetics or replaced teeth were consider by three of the four assessments. Patient oral hygiene was considered by two measures. The most contemporary approach included cosmetics and occlusal assessments, although many of its components occurred infrequently. At least for the present, then, a reasonable interpretation of clinical oral health status might include a summary measure of decayed, missing, and replaced teeth and periodontal pocketing or bone loss. Thus, we believed the OHSI adequately represents clinically observed oral health status and deserved to be evaluated.

Considering issues of validity again, construct validity involves assembling empirical evidence that implies the particular measure has meaning (6). This paper evaluates construct validity of the OHSI in relation to that of its individual components. That is, would any component of the index perform as well as or better than the index in its ability to differentiate among populations stratified by age, sex, race, education, income, self-assessments of general and oral health, preventive behaviors, and insurance status?

Hypotheses were generated for our expectations of the OHSI using a framework for health service utilization proposed by Andersen and others (15-17). This framework assumes that a sequence of conditions contributes to the extent that an individual will use health services (15). Use depends on the predisposition of an individual to use services; his or her ability to secure services (enabling conditions); and the individual's illness (or health) level, either perceived or evaluated. Illness level also has been characterized as being need, a biologic imperative to seek care or to consume services (16). Need then leads to use of services. The $\mathrm{OHSI}$, as a measure of evaluated oral health status, corresponds most closely to the illness level component of this model. Andersen also suggests that each component of the framework for health service utilization might be viewed as affecting subsequent components in the sequence, as well as making independent contributions to predicting use (16). In our paper, the framework is used as a general approach to assessing differences in oral health status. It is not intended to be a formal causal model or even a full listing of variables.

In this framework, predisposing factors include demographic, socialstructural, and attitudinal-belief variables. Of these factors, age, sex, race, and education were selected for the current analyses. We hypothesized, then, that OHSI scores would decline with age, be higher in females, be lower in nonwhites, and be higher in those with more education.

Enabling conditions make health services available to an individual, and include income, health insurance coverage, or other sources of third party payment, and whether or not an individual has a regular source of care (15). The current analyses used income and dental insurance status as enabling factors. For these factors, we hypothesized that OHSI scores would be better for those with higher incomes and those with dental insurance.

Perceived illness or oral health level was assessed using subjects' self-reported general health and oral health status. For these variables, we hypothesized that the OHSI would be positively correlated with self-reported general health and oral health status. Frequency of past checkups was considered a measure of historical use of services. OHSI scores were pre- dicted to be higher in those reporting more frequent dental checkups.

Two preventive behaviors - brushing and flossing - were included in our analyses because of relationships observed between these behaviors and periodontal health $(18,19)$. For these measures, we hypothesized that OHSI scores would be higher in individuals reporting acceptable brushing and flossing. Another behavior, smoking, was included because of the deleterious associations found between this activity and oral health (20-22). Subjects who smoked were hypothesized to have poorer OHSI scores than nonsmokers.

Additionally, hypotheses were generated for the ability of individual index components to perform similarly or better than the index as indicators of oral health status. Components were evaluated as mean unweighted (by index weights) counts of the various conditions. The missing tooth component was hypothesized to be most likely comparable to OHSI although operating inversely to the index. Thus, numbers of missing teeth were expected to rise with age, be more prevalent in nonwhites, decrease with rising education and income levels, decrease with self-reports of better general and oral health, and be lower in those subjects with acceptable preventive habits. The decayed tooth component was hypothesized to perform similarly to missing teeth with the exception that numbers of decayed teeth would decline with increasing age. We suspected that this inverse relation with age would "weaken" the performance of decayed teeth as a single measure of oral health status, at least compared to the OHSI or missing teeth.

The two periodontal components of the index were hypothesized to perform similarly to missing teeth; however, we expected these two components would be less strong as individual indicators of oral health status. The free end and replaced tooth components likely would vary similarly to the missing tooth component, but neither would be as strong an indicator of oral health status.

\section{Methods}

Oral Health Status Index. As proposed, the index is based on a clinical examination of a subject without radiographs. The examiner records a 
classification for each tooth: missing, replaced, decayed, or normal. A tooth is classified as missing if it is completely missing and an edentulous space is present. A tooth is classified as replaced if there is either a removable or fixed prosthetic replacement for it. If a tooth is absent and not replaced, and the remaining space measures $\leq 3$ millimeters, the space is regarded as being closed. This distinction allows differentiation between a missing tooth with space that may hinder function and a missing tooth with space closure that does not hinder function. A free end is defined as a quadrant missing at least all three molars.

A tooth is classified as decayed if there is gross cavitation, irregular discontinuity of the enamel's surface, or involvement of dentin. Incipient caries in pits and fissures of the tooth or demineralization of the enamel are not considered to be decay without dentin involvement.

A tooth is considered normal if it cannot be classified as missing, replaced, or decayed, or if it contains a sound restoration. Loss of periodontal attachment (LPA) is measured in the OHSI at the mesiofacial line angle of each tooth. Two categories of attachment loss are used with the index: sites with 4 or 5 millimeters of loss (moderate disease) or sites with $\geq 6$ millimeters of loss (severe disease). Extracted third molars are not counted as missing teeth, but are included when calculating free ends. Decay or loss of attachment on third molars is recorded if these conditions are observed.

To calculate an index score, the numbers of occurrences of the six conditions are totaled and multiplied by either a positive or negative coefficient. The component scores are added to 100 to derive a final score. The index was designed so that 100 equaled optimal health, and an edentulous patient with no replacements would receive a score of zero.

Study Population.The study population was a disproportionate probability sample of adults, 18 years of age or older living in housing units (e.g., single family houses, apartments, mobile homes) in the Detroit tricounty area. Sampling was done using stratified, clustered, area probability sampling techniques based on census tracts. For other analyses aimed at comparing African-Americans with whites and to separate the effects of race or ethnicity from socioeconomic status (SES), the sampling design was disproportionate, with AfricanAmericans being oversampled. One randomly selected adult was interviewed in each housing unit. This approach decreased dependence among subjects and undersampled persons living in larger households. Weights based on the sampling design corrected for the disproportionate sampling so that weighted results are representative of adults living in the Detroit tricounty area. SUDAAN was used to produce significance tests and variance estimates that accounted for intracluster correlations and disproportionate sampling of subjects.

Data were collected by face-to-face interviews performed all days of the week from May to September 1994. Interviewers made unlimited visits to each housing unit at varying times to maximize the chance of completing the interview. Interviews were conducted by professional interviewers trained both in general interviewing procedures and in use of the questionnaire. Questions were asked verbatim by the interviewers, with standardized probes as needed. The response rate for the interview was 71 percent $(n=787)$. At the time of the interview, subjects were invited to participate in the second phase of the study, a 50-minute in-home dental examination. Individuals who agreed to the dental examination are the subjects described in this report.

Oral Examination. Modified NIDR criteria (23) were used. Coronal and root surfaces on all teeth were examined for caries. Loss of attachment was measured at four sites (mesiofacial, facial, mesiolingual, distolingual) on all teeth. Our examination protocols were consistent with those of the OHSI and provided sufficient data for calculating the index. Four dentists calibrated on 12 subjects. Examiner agreement was assessed during the calibration sessions using Cohen's kappa (24). All possible pairings of examiners were evaluated. Kappa scores for coronal caries ranged from 0.88 to 0.97 . Kappa scores for loss of periodontal attachment $( \pm 1 \mathrm{~mm}$ ) ranged from 0.61 to 0.83 on lingual sites and 0.73 to 0.86 on facial sites.

Dentists sought and examined subjects from June through December 1994 any day or time during the week.
Informed consent was obtained from all participants, and a brief medical history was taken before the examination. Certain health conditions heart murmur, artificial heart valve, joint replacements - prevented the acquisition of complete examinations on some subjects. Subjects were paid $\$ 20$ or more if they completed the examination. Dental examinations were performed using available seating and head lamps. A total of 577 subjects was examined, 73.3 percent of those interviewed.

Data Analysis. Variables from the interview used in this analysis include subject's age, sex, race, income level, and education level. Subjects were asked whether they considered themselves primarily white or Caucasian, black or African-American, Asian or Pacific Islander, American Indian, Eskimo or Aleut, or other. For these analyses, subjects were categorized as nonwhite (black or African-American, Asian or Pacific Islander, American Indian, Eskimo or Aleut, or other) or white (white or Caucasian). Blacks or African-Americans comprised about 90 percent of the nonwhite group. The following age categorizations were used: 18 to 29 years old, 30 to 39 years old, 40 to 54 years old, and 55 years or older. Years of education were categorized as being under 12 years, 12 years, 13 to 15 years, or 16 years or older.

Family income was categorized as less than $\$ 20,000, \$ 20,000$ to $\$ 39,999$, $\$ 40,000$ to $\$ 69,999$, or $\$ 70,000$ or more. Subjects who had any dental insurance other than Medicaid (or in addition to Medicaid) were coded as dental insurance $=1$, all other subjects $=0$. Subjects who reported having only Medicaid were coded as Medicaid=1, all other subjects $=0$. This coding created a reference group of subjects with no dental insurance of any kind (no dental insurance, no Medicaid). Subjects were asked to assess their general health status using a five-point scale (excellent $=5$, very good $=4$, good $=3$, fair $=2$, poor $=1$ ) and their oral health status using a four-point scale (excellent $=4$, good $=3$, fair $=2$, poor $=1$ ).

Brushing, flossing, and dental checkup frequencies were acquired during the interview by asking subjects how often they performed these activities. Brushing and flossing variables were refined by including measures of thoroughness developed from previous investigations $(18,19)$. To be 
considered as having acceptable brushing behavior, a subject had to brush at least once daily during the past year and brush all his or her teeth. A subject who did not report performing the two aspects of brushing was considered to have unacceptable brushing behavior. For acceptable flossing behavior, a subject had to floss at least once daily during the past year and usually floss all of his or her teeth. A subject who did not report performing these two aspects of flossing was considered to have unacceptable flossing behavior.

Checkups were defined for subjects as visits to a dental care provider made not because of any dental problem. A checkup could include a cleaning, an examination, or both, and also could include treatment of dental problems that were discovered in the checkup. Subjects then were asked whether they had ever had a checkup, and how often they had gone to a dentist or dental clinic for a checkup during the past five years. Responses were dichotomized as those subjects having checkups at least once a year and those having checkups less frequently than once a year or never.

Bivariate relationships of all variables with OHSI scores and with index components were assessed with ANOVA using a significance level of $p<.05$. Correlation coefficients also were developed for all relationships. Then, multiple linear regression was used to identify associations between all independent variables and the sample's oral health status index score, and between the variables and the in- dividual components of the index.

For the correlation coefficients and the regression analyses, race was coded as nonwhite $=1$, white $=0$. Sex was coded as female $=1$, male $=0$. Selfreported brushing and flossing were coded as $1=$ acceptable behavior, $0=$ unacceptable behavior. Checkup frequency was coded as $1=$ at least one checkup per year, $0=$ checkups less than once a year. Smoking habits were coded as subject smokes $=1$, subject does not smoke $=0$. All other measures in the regression analyses were entered as continuous variables.

\section{Results}

Of the 787 subjects who completed an interview, $577(73.3 \%)$ were examined. Weighting the data to account for the disproportionate sampling re-

TABLE 1

Means and Standard Errors for Oral Health Status Index Scores and Index Components by Predisposing and Enabling Variables

\begin{tabular}{|c|c|c|c|c|c|c|c|c|}
\hline & \multirow[b]{2}{*}{$n$} & \multirow[b]{2}{*}{ OHSI } & \multicolumn{2}{|c|}{ Loss of Perio. Attachment } & \multicolumn{3}{|c|}{ Teeth } & \multirow[b]{2}{*}{ Free Ends } \\
\hline & & & $4-5 \mathrm{~mm}$ & $\geq 6 \mathrm{~mm}$ & Decayed & Missing & Replaced & \\
\hline \multicolumn{9}{|l|}{ Age (years) } \\
\hline $18-29$ & 122 & $94.5(1.09)^{*}$ & $.12(0.08)^{*}$ & $.05(0.04) \ddagger$ & $2.61(0.50) \ddagger$ & $.18(0.07)^{*}$ & $.01(0.01)^{*}$ & $.00(0.00)^{*}$ \\
\hline $20-39$ & 108 & $88.8(1.69)$ & $.59(0.27)$ & $.17(0.13)$ & $2.59(0.35 \ddagger$ & $1.77(0.35)$ & $.68(0.21)$ & $.15(0.04)$ \\
\hline $40-54$ & 146 & $82.4(3.17)$ & $.62(0.14)$ & $.34(0.14)$ & $1.56(0.29)$ & $4.25(0.77)$ & $2.53(0.60)$ & $.52(0.14)$ \\
\hline$\geq 55$ & 131 & $46.1(3.86)$ & $1.47(0.24)$ & $.68(0.20)$ & $1.15(0.16)$ & $15.87(1.30)$ & $13.62(1.30)$ & $2.35(0.20)$ \\
\hline \multicolumn{9}{|l|}{ Sex } \\
\hline Male & 232 & $78.9(1.65)$ & $.98(0.16) \ddagger$ & $.43(0.06)$ & $2.08(0.31)$ & $4.93(0.59)$ & $3.42(0.60)$ & $.64(0.09)$ \\
\hline Female & 277 & $75.9(2.62)$ & $.50(0.09)$ & $.23(0.10)$ & $1.80(0.22)$ & $6.42(0.84)$ & $5.21(0.82)$ & $.91(0.13)$ \\
\hline \multicolumn{9}{|l|}{ Race } \\
\hline White & 367 & $80.4(1.99)^{*}$ & $.69(0.12)$ & $.27(0.06)$ & $1.62(0.28) \ddagger$ & $5.02(0.63) \ddagger$ & $4.08(0.67)$ & $.69(0.10) \ddagger$ \\
\hline Nonwhite & 142 & $69.3(2.45)$ & $.77(0.14)$ & $.45(0.17)$ & $2.72(0.24)$ & $7.61(0.80)$ & $5.22(0.84)$ & $1.05(0.13)$ \\
\hline \multicolumn{9}{|l|}{ Education } \\
\hline$<12$ years & 79 & $52.8(5.01)^{*}$ & $.60(0.12)+$ & $.66(0.36)+$ & $2.39(0.56)^{*}$ & $13.17(1.69)^{*}$ & $10.11(1.72)^{*}$ & $1.75(0.30)^{*}$ \\
\hline 12 years & 166 & $72.8(2.41)$ & $1.05(0.19)$ & $.39(0.15)$ & $2.26(0.28)$ & $6.76(0.88)$ & $5.32(0.94)$ & $1.05(0.15)$ \\
\hline $13-15$ years & 128 & $85.4(2.21)$ & $.45(0.16)$ & $.14(0.06)$ & $2.15(0.34)$ & $3.20(0.68)$ & $2.05(0.61)$ & $.39(0.10)$ \\
\hline$\geq 16$ years & 134 & $89.6(1.70)$ & $.63(0.12)$ & $.21(0.08)$ & $1.03(0.21)$ & $2.51(0.42)$ & $2.10(0.37)$ & $.28(0.06)$ \\
\hline \multicolumn{9}{|l|}{ Income } \\
\hline$<\$ 20,000$ & 113 & $67.8(3.72)^{*}$ & $.84(0.13)$ & $.47(0.17)$ & $3.20(0.37)^{*}$ & $7.82(1.38)^{*}$ & $5.55(1.49)^{*}$ & $1.08(0.22)$ \\
\hline$\$ 20 \mathrm{~K}-39,999$ & 111 & $68.5(3.84)$ & $.60(0.14)$ & $.30(0.09)$ & $2.00(0.33)$ & $8.62(1.34)$ & $6.98(1.39)$ & $1.23(0.22)$ \\
\hline$\$ 40 \mathrm{~K}-69,999$ & 152 & $81.5(2.92)$ & $.82(0.29)$ & $.35(0.15)$ & $1.69(0.35)$ & $4.45(0.80)$ & $3.27(0.72)$ & $.61(0.14)$ \\
\hline$\geq \$ 70,000$ & 131 & $88.2(2.02)$ & $.58(0.15)$ & $.18(0.09)$ & $1.05(0.23)$ & $3.00(0.54)$ & $2.51(0.46)$ & $.36(0.10)$ \\
\hline \multicolumn{9}{|l|}{$\begin{array}{l}\text { Dental } \\
\text { insurance }\end{array}$} \\
\hline Yes & 344 & $79.8(1.64) \dagger$ & $.70(0.10)$ & $.26(0.08)$ & $1.55(0.22)^{*}$ & $5.20(0.55)$ & $4.03(0.55)$ & $.73(0.10)$ \\
\hline Medicaid & 30 & $72.8(4.90)$ & $1.09(0.45)$ & $.68(0.45)$ & $4.63(0.61)$ & $5.07(1.15)$ & $2.59(0.68)$ & $.56(0.16)$ \\
\hline No & 133 & $71.6(4.09)$ & $.67(0.18)$ & $.40(0.14)$ & $2.30(0.36)$ & $7.33(1.32)$ & $5.78(1.26)$ & $1.00(0.20)$ \\
\hline
\end{tabular}

${ }^{*} P<.001$

$+P<05$.

$\ddagger P<.01$. 
sulted in a final sample of 570 , of whom 509 completed enough of the examination to calculate an OHSI score. The numbers of subjects in each table represent weighted sample sizes.

The mean OHSI score for the 509 subjects was 77.3 ( $\mathrm{SE}=1.83$ ) with a range of -8.0 to 100.0 . The 25 th percentile was 67.8 , the 50 th percentile was 90.8 , and the 75 th percentile was 98.2. About one out of five subjects (21.6\%) had an OHSI score of 100.0. A smaller group of edentulous subjects clustered at the lower end of the range. Of the edentulous subjects, one had no tooth replacements (OHSI $=0.00)$, three had only a single denture (mean OHSI= 8.4 ), and 45 subjects had complete dentures (mean $\mathrm{OHSI}=16.9$ ).

Table 1 displays mean OHSI and index component scores by predisposing and enabling variables. Differences in index scores among the four age groups were statistically signifi- cant $(P<.001)$. OHSI scores progressively declined in the first three age groups, then dropped precipitously in the oldest age group. As can be observed in the average counts of conditions (index components), the drop in OHSI was primarily related to increased loss of teeth with associated free ends. The mean replaced tooth component was greatest in the oldest age group as was attachment loss of 4-5 $\mathrm{mm}$ or $\geq 6 \mathrm{~mm}$. Conversely, the mean number of decayed teeth was largest in the youngest age group, then declined in successively older groups.

No statistically significant difference in OHSI scores for males and females was observed. The only significant difference in component scores was found to be more loss of attachment $(4-5 \mathrm{~mm})$ in males. The difference in oral health status scores between races was statistically significant $(P<.001)$, with nonwhites having a lower score than whites. In the component scores, more decayed teeth, missing teeth, and free ends were observed in nonwhites.

A 37-point difference in OHSI scores was observed between the lowest and highest education level $(P<.001)$. All index components demonstrated statistically significant differences by education level. Subjects reporting less than 12 years of education had more loss of attachment $(\geq 6$ $\mathrm{mm}$ ); more decayed, missing, and replaced teeth; and more free ends. Generally, components declined in magnitude with increasing education level.

OHSI scores improved as income level increased $(P<.001)$. The component scores that appeared to contribute most to overall index scores by income level were decayed, missing, and replaced teeth. Subjects having any dental insurance other than Medicaid (collectively called dental insur-

TABLE 2

Means and Standard Errors for Oral Health Status Index Scores and Index Components by Self-reported General and Oral Health Status and Selected Behaviors

\begin{tabular}{|c|c|c|c|c|c|c|c|c|}
\hline & \multirow[b]{2}{*}{$n$} & \multirow[b]{2}{*}{ OHSI } & \multicolumn{2}{|c|}{ Loss of Perio. Attachment } & \multicolumn{3}{|c|}{ Teeth } & \multirow[b]{2}{*}{ Free Ends } \\
\hline & & & 4-5 mm & $\geq 6 \mathrm{~mm}$ & Decayed & Missing & Replaced & \\
\hline \multicolumn{9}{|l|}{$\begin{array}{l}\text { General health } \\
\text { (self) }\end{array}$} \\
\hline Excellent & 123 & $91.7(1.35)^{*}$ & $.48(0.13)$ & $.15(0.09) \dagger$ & $1.22(0.15) \dagger$ & $1.70(0.35)^{*}$ & $.91(0.34)^{*}$ & $.17(0.05)^{*}$ \\
\hline Very good & 226 & $79.4(2.15)$ & $.72(0.15)$ & $.33(0.10)$ & $2.00(0.33)$ & $5.06(0.62)$ & $4.01(0.61)$ & $.68(0.10)$ \\
\hline Good & 113 & $70.8(4.30)$ & $.99(0.15)$ & $.25(0.07)$ & $2.56(0.30)$ & $7.39(1.26)$ & $5.50(1.06)$ & $1.08(0.19)$ \\
\hline Fair/poor & 40 & $42.9(7.55)$ & $.64(0.18)$ & $1.00(0.64)$ & $1.98(0.47)$ & $16.20(2.38)$ & $12.90(2.34)$ & $2.32(0.40)$ \\
\hline \multicolumn{9}{|l|}{ Oral health (self) } \\
\hline Excellent & 102 & $85.7(2.62)^{*}$ & $.23(0.10) \dagger$ & $.02(0.01)^{*}$ & $.62(0.17)^{*}$ & $4.28(0.87) \dagger$ & $3.79(0.90)$ & $.61(0.13) \dagger$ \\
\hline Good & 253 & $81.0(2.60)$ & $.71(0.12)$ & $.27(0.08)$ & $1.40(0.12)$ & $4.98(0.87)$ & $4.19(0.84)$ & $.67(0.13)$ \\
\hline Fair & 130 & $65.9(3.55)$ & $.94(0.24)$ & $.50(0.17)$ & $3.40(0.40)$ & $8.20(1.08)$ & $5.61(1.08)$ & $1.15(0.17)$ \\
\hline Poor & 23 & $63.6(6.35)$ & $1.67(0.48)$ & $1.23(0.43)$ & $5.16(0.44)$ & $6.68(1.66)$ & $2.49(1.40)$ & $.91(0.31)$ \\
\hline \multicolumn{9}{|l|}{ Brushing habits } \\
\hline Unacceptable & 45 & $84.3(3.03)$ & $.44(0.18) \neq$ & $.25(0.13)$ & $3.18(1.05)$ & $2.80(0.71)$ & $1.32(0.42)$ & $.31(0.12)$ \\
\hline Acceptable & 418 & $83.3(1.62)$ & $.82(0.09)$ & $.36(0.07)$ & $2.01(0.21)$ & $3.60(0.42)$ & $2.25(0.33)$ & $.49(0.07)$ \\
\hline \multicolumn{9}{|l|}{ Flossing habits } \\
\hline Uncceptable & 378 & $83.6(1.58)$ & $.75(0.09)$ & $.29(0.07)$ & $2.23(0.23) \ddagger$ & $3.50(0.41)$ & $2.24(0.34)$ & $.46(0.08)$ \\
\hline Acceptable & 84 & $82.6(2.91)$ & $.97(0.29)$ & $.63(0.28)$ & $1.53(0.32)$ & $3.67(0.69)$ & $1.84(0.66)$ & $.53(0.15)$ \\
\hline \multicolumn{9}{|l|}{ Checkups/5-year } \\
\hline$<1 /$ yr or never & 173 & $64.0(3.04)^{*}$ & $.94(0.19)$ & $.50(0.14)$ & $2.83(0.37) \dagger$ & $9.31(1.19)^{*}$ & $7.39(1.28)^{*}$ & $1.29(0.19) \dagger$ \\
\hline At least once/yr & 336 & $84.1(1.28)$ & $.60(0.07)$ & $.23(0.05)$ & $1.46(0.20)$ & $3.90(0.37)$ & $2.85(0.32)$ & $.53(0.07)$ \\
\hline \multicolumn{9}{|l|}{ Subject smokes } \\
\hline No & 347 & $78.0(2.72)$ & $.65(0.07)$ & $.28(0.09)$ & $1.50(0.14)^{*}$ & $5.83(0.90)$ & $4.67(0.89)$ & $.82(0.14)$ \\
\hline Yes & 163 & $75.8(1.85)$ & $.86(0.22)$ & $.40(0.10)$ & $2.83(0.32)$ & $5.56(0.62)$ & $3.81(0.62)$ & $.71(0.10)$ \\
\hline
\end{tabular}

${ }^{*} P<.001$.

tP<.01.

$\ddagger P<05$. 
TABLE 3

Correlation Coefficients for Independent Variables in Relation to Oral Health Status Index and Index Components

\begin{tabular}{|c|c|c|c|c|c|c|c|}
\hline & \multirow[b]{2}{*}{ OHSI } & \multicolumn{2}{|c|}{ Loss of Perio. Attachment } & \multicolumn{3}{|c|}{ Teeth } & \multirow[b]{2}{*}{ Free Ends } \\
\hline & & 4-5 mm & $\geq 6 \mathrm{~mm}$ & Decayed & Missing & Replaced & \\
\hline Age & $-.64^{*}$ & $.24^{*}$ & $.18 \ddagger$ & $-.23^{*}$ & $.66^{*}$ & $.60^{*}$ & $.63^{*}$ \\
\hline Sex & -.05 & $-.13 t$ & -.08 & -.05 & .08 & .01 & .09 \\
\hline Race & $-.17^{*}$ & .02 & .06 & $.17^{*}$ & $.13 \ddagger$ & .06 & $.11 \ddagger$ \\
\hline Education & $.44^{*}$ & -.03 & -.12 & $-.14 t$ & $-.40^{*}$ & $-.32^{*}$ & $-.37^{*}$ \\
\hline Income & $.25^{*}$ & -.05 & $-.08+$ & $-.24 \ddagger$ & $-.19^{*}$ & $-.13 t$ & $-.19 \ddagger$ \\
\hline Dental insurance & $.13 t$ & -.01 & -.07 & $-.19 \ddagger$ & -.09 & -.06 & -.06 \\
\hline Medicaid & -.04 & .05 & .07 & $.23^{*}$ & -.02 & -.05 & -.04 \\
\hline General health & $.42^{*}$ & -.06 & -.13 & $-.13 \ddagger$ & $-.38^{*}$ & $-.31^{*}$ & $-.36^{*}$ \\
\hline Oral health & $.26^{*}$ & $-.17 \ddagger$ & $-.19 \ddagger$ & $-.41^{*}$ & $-.14 t$ & -.04 & $-.12 t$ \\
\hline Brushing & -.01 & $.06 t$ & .03 & -.12 & .03 & .03 & .04 \\
\hline Flossing & -.01 & .05 & .10 & $-.09+$ & .01 & -.02 & .02 \\
\hline Checkups & $.33^{*}$ & -.09 & -.10 & $-.22 \ddagger$ & $-.28^{*}$ & $-.24 \ddagger$ & $-.25 \ddagger$ \\
\hline Smoking & -.04 & .05 & .04 & $.21^{*}$ & -.01 & -.05 & -.03 \\
\hline
\end{tabular}

${ }^{*} P<, 001$.

$+P<.05$.

$\ddagger P<.01$.

ance) had a mean OHSI score of about 80 , while subjects who reported having only Medicaid or no dental insurance had mean OHSI scores of about 72. For the Medicaid group, caries, periodontal condition, and fewer replaced teeth contributed to their low OHSI scores, while missing teeth and free ends helped to produced low OHSI scores in those with no dental insurance.

Table 2 exhibits mean OHSI and component scores by self-reported general and oral health status, preventive behaviors, and smoking habits. OHSI scores declined as subjects' selfreports of general health status worsened. About a 50-point difference was observed between those reporting excellent health and those with fair/poor general health. Statistically significant differences were observed in all component scores except 4-5 mm LPA. These trends are similar to those observed with age, a finding that is not surprising because of the likely relationship of poorer self-assessed health as age increases. An exception is the decayed tooth component that increases as self-assessed health status declines until the category fair/poor. The mean number of decayed teeth in this category likely is lower because many teeth are missing. Similarly, OHSI scores declined as subjects' reported poorer oral health status $(P<.001)$. All component values in- crease as self-reported oral health status changed from excellent to good to fair. The small group ( $n=23$ ) reporting poor oral health status reversed the trends of increasing missing and replaced teeth; however, these subjects were younger on average than the other groups.

Of the preventive behaviors and prior service utilization that were assessed (brushing, flossing, and checkups), only checkups demonstrated a statistically significant difference in OHSI scores $(P<.001)$. All index component scores except $4-5 \mathrm{~mm}$ and $\geq 6$ mm LPAs were also statistically different for checkup frequency, with those reporting more frequent checkups having fewer decayed, missing, and replaced teeth and free ends. No statistically significant difference in OHSI scores was observed between subjects who smoked and those who did not. For component scores, smokers had significantly more decayed teeth.

Table 3 shows the correlation coefficients for the independent variables in relationship to the OHSI scores and index component values. Of all the index components, decayed teeth and missing teeth often demonstrated similar or stronger statistically significant correlations to the independent variables than did the OHSI.

As for strength of correlations, income, education, self-assessed general health, and checkup frequency were more strongly correlated with the OHSI than with any of its components. Race was correlated most strongly (0.17) with OHSI and decayed teeth. Self-assessed oral health, flossing, having dental insurance, having Medicaid, and smoking were more strongly correlated with decayed teeth than with OHSI or any other component. Age was more strongly correlated with missing teeth than with OHSI; however, the coefficients were quite similar. Although they never had the strongest correlations, replaced teeth and free ends had many substantial correlations that were generally of the same sign and magnitude as correlations of missing teeth.

Table 4 displays the final models for determining correlates of OHSI and index components using linear regression. Better oral health status as measured by the OHSI was associated with younger individuals, white race, higher education, better self-assessed oral health status, more frequent checkups, and not smoking. About 53 percent of the variance in OHSI scores was explained by the model. Of the index components, the model for missing teeth was most successfully predicted with 46 percent of the variance being explained. Fewer missing teeth (better oral health) were associated with younger individuals, white race, higher education, better self-re- 
ported oral health, having Medicaid, and not smoking.

About 36 percent of the variance was explained by the model for free ends, with fewer free ends likely to be observed in young individuals, males, white race, higher income individuals, those with better self-assessed oral health, and those with Medicaid. Only 28 percent of the variance was explained by the model for decayed teeth. In this model, younger age, higher incomes, better self-assessed oral health, and not smoking were associated with having fewer decayed teeth.

\section{Discussion}

The goals of this investigation were to evaluate the OHSI and to determine if any index component might perform as well as or better than the overall index as an indicator of oral health status. The observed patterns of OHSI scores in this research appeared to provide some support for its construct validity.

OHSI scores declined with age, thus confirming our hypothesis. In the final regression models, age was a negative correlate of the OHSI, but demonstrated positive correlations in the models for all other components except decayed teeth. The inverse relation of decayed teeth with age makes it somewhat problematic to use decayed teeth as a sole indicator of oral health status or to combine it with another indicator such as missing teeth. However, the OHSI accommodated these "opposing" contributions to poor oral health status by negatively weighting counts of its detrimental components.

Our hypothesis that females would have better OHSI scores than males was not supported. Sex did not prove to be a statistically significant correlate of OHSI in the regression analyses. Sex was a significant correlate in regression models for some components of the index. For example, it was associated with moderate (4-5 mm LPA) and severe ( $\geq 6 \mathrm{~mm}$ LPA) attachment loss. Male subjects were more likely to have greater attachment loss, which is consistent with the notion that sex is a correlate of attachment loss (25). Thus, use of the complete index masked this relationship.

The last two predisposing variables, race and education, performed similarly in the regression analyses, being correlates of the OHSI and missing teeth. Nonwhite race was also a positive correlate in the model for free ends. In all three models, these independent variables operated consistently with our expectations and with observations by Chen (26). She re- viewed the oral health status of disadvantaged populations, defined as groups with low socioeconomic status (SES) or minorities. Low SES adults were found to have more untreated decayed teeth, more missing teeth, and more edentulousness. Generally, the dentition of adults in minority groups also was found to be poorer than that of whites. Another component of SES - income - and one of our enabling variables - remained in the regression models for decayed teeth and free ends. Disadvantaged populations often use Medicaid as a payment source for dental care (27). In our analyses, Medicaid was a correlate of missing teeth, replaced teeth, and free ends. In sum, missing teeth or free ends might be useful measures of oral health status in disadvantaged populations.

In the regression models, self assessment of oral health was statistically significant for OHSI, 4-5 mm LPA, decayed teeth, missing teeth, and free ends. These findings provide some empirical evidence that the OHSI and these particular components might be measuring oral health in a manner that is consistent with individuals' perceptions of that status. While not specifically supporting criterion validity of the index, self-reported status is another observation

TABLE 4

Standardized Regression Coefficients for Independent Variables on OHSI and Index Components, and Overall Model $\mathbf{R}^{2}$

\begin{tabular}{|c|c|c|c|c|c|c|c|}
\hline & OHSI & $\begin{array}{c}4-5 \mathrm{~mm} \\
\text { LPA }\end{array}$ & $\begin{array}{c}\geq 6 \mathrm{~mm} \\
\text { LPA }\end{array}$ & $\begin{array}{l}\text { Decayed } \\
\text { Teeth }\end{array}$ & $\begin{array}{l}\text { Missing } \\
\text { Teeth }\end{array}$ & $\begin{array}{l}\text { Replaced } \\
\text { Teeth }\end{array}$ & $\begin{array}{l}\text { Free } \\
\text { Ends }\end{array}$ \\
\hline Age & $-.48^{*}$ & $.46^{*}$ & $.31^{*}$ & $-.10+$ & $.43^{*}$ & $.31^{*}$ & $.40^{*}$ \\
\hline Sex & -.01 & $-.14+$ & $-.12+$ & -.05 & .05 & $.08+$ & $.06 t$ \\
\hline Race & $-.14^{*}$ & .05 & .07 & .09 & $.10^{*}$ & .03 & $.08+$ \\
\hline Education & $.09+$ & .08 & -.08 & -.05 & $-.07+$ & -.03 & -.05 \\
\hline Income & .05 & -.02 & .04 & $-.07 t$ & -.04 & -.03 & $-.08 t$ \\
\hline Dental insurance & .05 & .04 & -.05 & -.06 & -.04 & -.02 & -.01 \\
\hline Medicaid & .03 & .11 & .04 & .09 & $-.05 \ddagger$ & $-.05 t$ & $-.07 t$ \\
\hline General health & .03 & $.09+$ & .01 & -.02 & -.03 & -.05 & -.05 \\
\hline Oral health & $.19^{*}$ & $-.20+$ & -.18 & $-.30^{*}$ & $-.10 \dagger$ & -.02 & $-.08+$ \\
\hline Brushing & -.02 & $.08+$ & .04 & -.05 & .02 & .02 & .03 \\
\hline Flossing & .00 & .04 & .12 & -.03 & -.02 & -.05 & -.01 \\
\hline Checkups & $.08+$ & -.10 & -.06 & -.09 & -.06 & -.05 & -.05 \\
\hline Smoking & $-.09 \ddagger$ & .09 & .04 & $.09+$ & $-.07 \dagger$ & .04 & .04 \\
\hline $\mathrm{R}^{2}$ & .53 & .21 & .15 & .28 & .046 & .29 & .36 \\
\hline
\end{tabular}

${ }^{*} P<.001$

$+P<05$.

$\neq P<01$. 
about oral health; both measures were congruent in direction.

Of the preventive behaviors, only checkup frequency remained as a correlate of OHSI in the regression analyses. Checkup frequency has been associated with better oral health $(18,28)$; and the positive relationship between OHSI scores and checkup frequency supports this premise. A detrimental behavior, smoking, was negatively correlated with OHSI scores and positively correlated with decayed and missing teeth when other factors were controlled. These relationships also are consistent with our expectations.

From these analyses, it would seem that one could choose different representations of oral health status depending on what, where, and for whom measurements were being performed. Assessments could range from a single item, multiple items, or an index such as the OHSI. If, for example, a public program had limited resources for measurement, then counting missing teeth or free ends might be sufficient indicators of clinical status. Then, subjects could be asked to evaluate their own oral health using the single item described previously. Perhaps missing teeth and self-assessed oral health could be combined in some manner with weighting to form a simple composite measure.

Where need or opportunity for more extensive data collection exists, calculating the OHSI as an additional indicator of oral health status might be appropriate. The oral conditions used historically in composite indices caries, periodontal disease, missing teeth - are included in the OHSI, as is a replaced teeth component. The correlations of the OHSI with independent variables used in these analyses were generally stronger than any single component, and more variance was explained by the OHSI regression model than any other. Thus, it seems the OHSI may be useful in certain settings.

From a practical perspective, the OHSI was simple to use. In this investigation, the necessary measurements were collected as part of a more extensive examination lasting 50 minutes. Performing only the OHSI would have shortened the examination. The index was easily quantifiable. Conceivably, scores could be generated and graphically viewed at chairside by a patient.

While the OHSI can provide a clini- cal measure of health status, it does not address behavioral or subjective consequences of oral conditions such as impairment, functional limitation, discomfort, disability, or handicap as described by Locker (29). Subjective selfassessments vary and have been composed of a single item (30), a few items $(31,32)$, or many items $(33)$. Thus, one or several items could be used in addition to a clinical measure. Contemporary thinking on assessment of oral health status requires that subjective and patient-centered measures be used; however, it remains to be determined whether they will function best as independent, but equal, assessments or in some composite measure that contains clinical and subjective measures.

As previously noted, the OHSI was constructed nearly 20 years ago, and may not represent current thinking about index components or construction (3). While newer indices may be available, none have been reported in the published literature. Thus, it remains to be seen if the OHSI contains the "right" number and kinds of components. Other components that might be considered include some measure of oral hygiene (10) or plaque or gingival bleeding measures (Sainfort $F$, Zimmerman DR, Booske BC, Wickeham D. Oral health outcomes measurement using multiattribute utility theory. Unpublished manuscript, 1994). Conditions such as oral pathology, or temporomandibular dysfunction might be incorporated into an index; but these conditions appear to occur infrequently.

If index components are to be weighted, the prevalence of a condition and its contribution to oral health status should be determined. If a condition is observed infrequently but is severe if present, a weighting scheme that reflects this situation would be useful. However, a more practical approach might be to exclude some items from an index if they occur infrequently to simplify the overall measure. Tooth loss likely will be included in any index; however, it is a condition whose weighting may need to change over time. If weights were changed to reflect contemporary disease trends, older weight sets could be applied to facilitate retrospective comparisons.

Given our findings regarding the OHSI, it has many elements that make it appealing as a foundation for contin- ued work on composite measures of oral health status. It has components encompassing contemporary oral diseases and conditions; it weights these components such that a higher score is indicative of better oral health; it has few components, which makes it is easy to apply and interpret; it appears to have construct validity; and it is correlated with a rudimentary, singleitem measure of patient self-assessed oral health. Continued evaluation of its utility is warranted, and understanding its relationship to patients' self-assessed oral health deserves study.

\section{References}

1. Burt B, Eklund S. Dentistry, dental practice and the community. Philadelphia: Saunders, 1992.

2. Dunning J. Principles of dental public health. Cambridge: Harvard University Press, 1979.

3. Coulter ID, Marcus M, Atchison KA Measuring oral health status: theoretical and methodological challenges. Soc $\mathrm{Sci}$ Med 1994;38:1531-41.

4. Reisine S. An overview of self-reported outcome assessment in dental research. J Dent Educ 1996;60:488-93.

5. Marcus M, Koch AL, Gershen JA. A proposed index of oral health status: a practical application. J Am Dent Assoc 1983; 107:729-33.

6. Kaplan RM, Bush JW, Berry CC. Health status: types of validity and the index of well-being. Health Serv Res 1976;11:478507.

7. Marcus M, Koch A, Gershen J. Construction of a population index of adult oral health status derived from dentists' preferences. J Public Health Dent 1983;43: 28494.

8. Bulman J. Demand and need for dental care; a socio-dental study. London: $\mathrm{Ox}$ ford University Press, 1968.

9. Nikias M, Sollecito W, Fink R. An empirical approach to developing multidimensional oral status profiles. J Public Health Dent 1978:38:148-58.

10. Nikias $M$, Sollecito $W$, Fink R. An oral health index based on ranking oral health profiles by panels of dental professionals. J Public Health Dent 1979:39:16-26.

11. Koch AL. Application of a paired preference computer scaling technique to model expert decision making for the development of an oral health status index. Los Angeles: University of California, 1981.

12. Marcus M, Koch A, Gershen J. An empirically derived measure of oral health status for adult populations. J Public Health Dent 1980;40:334-45.

13. Marcus M, Gershen J, Koch A. Indirect and direct adult oral health status index user's guide. Development of oral health status measures for quality assurance. Los Angeles: University of California, 1980.

14. Braun RJ. A methodology for a pilot study to develop a geriatric oral health 
status index. J Dent Educ 1985:49:753-6.

15. Andersen R, Newman JF. Societal and individual determinants of medical care utilization in the United States. Milbank Mem Fund Q Health Soc 1973;51:95-124.

16. Andersen RM. Revisiting the behavioral model and access to medical care: does it matter? J Health Soc Behav 1995;36:1-10.

17. Aday LA, Andersen R. A framework for the study of access to medical care. Health Serv Res 1974;9:208-20.

18. Lang WP, Ronis DL, Farghaly MM. Preventive behaviors as correlates of periodontal health status. J Public I lealth Dent 1995;55:10-17.

19. Lang WP, Farghaly MM, Ronis DL. The relation of preventive dental behaviors to periodontal health. J Clin Periodontol 1994:21:194-8.

20. Norlen P, Ostberg H, Bjorn AL. Relationship between general health, social factors and oral health in women at the age of retirement. Community Dent Oral Epidemiol 1991;19:296-301.

21. Eklund SA, Burt BA. Risk factors for total tooth loss in the United States; longitudinal analysis of national data. J Public
Health Dent 1994:54:5-14.

22. Locker D. Smoking and oral health in older adults. Can J Public Health 1992;83: 429-32.

23. Miller AJ, Brunelle JA, Carlos JP, Brown LJ, Löe H. Oral health of United States adults. The national survey of oral health in US employed adults and seniors: 1985 1986. National findings. Washington, DC: US Government Printing Office, US Department of Health and Human Services, Public Health Service, 1987.

24. Hunt R. Percent agreement, Pearson's correlation, and kappa as measures of inter-examiner reliability. J Dent Res 1986;65:128-30.

25. American Academy of Periodontology's Research, Science, and Therapy Committee. Epidemiology of periodontal diseases. J Periodontol 1996;67:935-45.

26. Chen MS. Oral health of disadvantaged populations. In: Cohen L, Gift H, eds. Disease prevention and oral health promotion. Copenhagen: Munksgaard, 1995:153-212.

27. Public Health Service's Oral Health Coordinating Committee. Toward improv- ing the oral health of Americans: an overview of oral health status, resources, and care delivery. Public Health Rep 1993; 108:657-72.

28. Frandsen A. Mechanical oral hygiene practices. State-of-the-science review. In: Löe H, Kleinman DV, eds. Dental plaque control measures and oral hygiene practices. Bethesda, MD: IRL Press, 1985:93116.

29. Locker D. Measuring oral health: a conceptual framework. Community Dent Health 1988;5:3-18

30. Reisine ST, Bailit HL. Clinical oral health status and adult perceptions of oral health. Soc Sci Med 1980;14A:597-605.

31. Gooch B, Dolan T, Bourque L. Correlates of self-reported dental health status upon enrollment in the Rand Heal th Insurance Experiment. J Dent Educ 1989;53:629-37.

32. Atchison K, Dolan T. Development of the Geriatric Oral Health Assessment Index. J Dent Educ 1990;54:680-7.

33. Slade GD, Spencer AJ. Development and evaluation of the Oral Health Impact Profile. Community Dent Health 1994;11:311. 